\title{
Suicide in patients with stroke: epidemiological study
}

\author{
Elsebeth Nylev Stenager, Claus Madsen, Egon Stenager, Jesper Boldsen
}

Department of Social Medicine, Odense

Municipality,

Vindegade 18, 5000

Odense C,

Denmark

Elsebeth Nylev

Stenager,

registrar

Department of

Neurology, Odense

University Hospital,

Odense, Denmark

Claus Madsen,

registrar

Department of

Neurology, Esbjerg

Centralsygehus,

Esbjerg, Denmark

Egon Stenager,

senior neurology

consultant,

Institute of

Community Health,

Odense University,

Winsløwsparken,

5000 Odense C,

Denmark

Jesper Boldsen,

associate professor

Correspondence to:

Dr Stenager

BMJ 1998;316:1206
In the United States stroke is the third most common cause of death among those aged over 70. In Europe the incidence of stroke is 2 per 1000 population a year. ${ }^{1}$ Survivors are often incapacitated. The frequency of depression after stroke is estimated at $18-60 \%{ }^{2}$ Other neurological disorders that may result in mental and cognitive disorders are associated with an increased risk of suicidal behaviour. ${ }^{3}$ Studies on the possible increased risk of suicide among patients with stroke have never been performed. We therefore aimed to estimate, on the basis of a cohort of patients with stroke in a selected area in Denmark, whether the risk of suicide was higher than in the background population (the common reference in previous studies on suicide risk ${ }^{3}{ }^{4}$.

\section{Materials, method, and results}

All patients admitted to hospital in the county of Funen, Denmark, with a discharge diagnosis of stroke (code 430-438 according to the international classification of diseases, eighth revision) during 1 April 1973 to 31 December 1989 were registered.

Only admitted patients were included, biasing the study towards the patients with the most severe strokes. Danish patients with stroke, however, are almost always admitted, thus reducing this bias.

Age, date of birth, sex, time of first admittance, and department of admittance were registered. In patients who had died, the date of death was also registered. Information was collected for the study period from the National Board of Health on (a) the causes of death in the deceased patients, and $(b)$ the frequency of suicide comparable for age and sex in the total population of the county of Funen (the background population). We then calculated standardised mortality ratios for suicide for men and women separately in the age groups $\leqslant 49$ years, 50-59, 60-69, 70-79, and $\geqslant 80$. The study was approved by the regional ethics committee for the county of Funen and Vejle and the Danish Data Protecting Agency.

At the end of the study, of the 37869 patients with stroke (19 266 men), 7365 (3614 men) were alive and 30504 (15652 men) had died. Altogether, 140 patients ( 80 women) committed suicide. The table shows the number of suicides, the person years at risk, and the standardised mortality ratios in the five age groups for women and men.

\section{Comment}

We have shown that patients with stroke have a significantly increased risk of suicide, especially in the age groups up to age 60 and in women.

The high suicide risk in the youngest age groups is in agreement with studies on suicide in multiple sclerosis, ${ }^{3}$ epilepsy, Huntington's chorea, spinal cord lesions, and diabetes. ${ }^{4}$ The lowered risk of suicide in the oldest age groups is also in agreement with the findings for Parkinson's disease. ${ }^{4}$

In multiple sclerosis, men had the highest suicide risk, whereas in stroke, women did. A Danish study found an increased risk of depression in female patients with stroke, ${ }^{5}$ which might explain the finding. Because we included all admitted patients with stroke in the area (possible because of good registration practice), selection bias could not explain the results. Furthermore, the large number of patients included in the study makes the results reliable.

Although only 140 in a population of almost 38000 patients with stroke committed suicide $(7.2 \%$ of all the suicides in the area), an unknown, but probably larger number of patients may have attempted suicide, and a third may have depression. ${ }^{2}$ Furthermore, the number of suicides may be underestimated as some deaths would not be registered as suicides. The high suicide risk in patients with stroke suggests that society should take more interest in the psychosocial aspects of living with the impairment imposed by stroke.

Contributors: CM, ENS, and ES wrote the project protocol. $\mathrm{CM}$ and ENS coordinated the collection of the data. ENS wrote the initial version of the paper, which was discussed and accepted by all authors. ES had the original idea for the study and participated in the discussion of the protocol and data sampling and in the discussion of the paper. $\mathrm{JB}$ was responsible for the data analysis and made all the data analyses. Afterwards the results were discussed by all the authors.

Funding: The study has received financial support from the Ministry of Health in Denmark.

Conflict of interest: None.

1 Robins M, Baum HM. Incidence: the national survey of stroke. Stroke. 1981;12(suppl 1):45-57.

2 House A, Dennis M,Mogridge L, Warlow C, Hawton K, Jones L. Mood disorders in the year after first stroke. Br J Psychiatry 1991;158:83-92.

3 Stenager EN, Stenager E. Suicide in patients with neurological diseases Methodological problems. Literature review. Arch Neurol 1992;49:1296303.

4 Stenager EN, Stenager E. Disease, pain and suicidal behaviour. New York: Haworth Press, 1997.

5 Andersen G, Vestergård K, Ingemann-Nielsen M, Lauritzen L. Risk factors for post stroke depression. Acta Psychiatr Scand 1995;92:193-8. (Accepted 4 December 1997)

Number of suicides and standardised mortality ratios (95\% confidence interval) for suicide in women and men with stroke in five age groups

\begin{tabular}{lccccccc} 
& \multicolumn{2}{c}{ Women } & & \multicolumn{2}{c}{ Men } \\
\cline { 2 - 4 } \cline { 6 - 8 } Age group & Suicides & Years at risk & Standardised mortality ratio & & Suicides & Years at risk & Standardised mortality ratio \\
\hline$\leqslant 49$ & 7 & 1964.2 & $1376(646$ to 2996$)$ & & 6 & 2771.1 & $656(324$ to 1352$)$ \\
\hline $50-59$ & 22 & 4096.3 & $1378(896$ to 2129$)$ & & 20 & 7514.8 & $580(338$ to 823$)$ \\
\hline $60-69$ & 7 & 11263.2 & $224(120$ to 426$)$ & & 9 & 15031.0 & $76(38$ to 157$)$ \\
\hline $70-79$ & 25 & 21481.6 & $184(112$ to 304$)$ & & 15 & 18680.2 & $161(98$ to 224$)$ \\
\hline$\geqslant 80$ & 19 & 17640.7 & $133(73$ to 244$)$ & & 10 & 9192.8 & $131(84$ to 205$)$
\end{tabular}

